INPLASY

PROTOCOL

To cite: Velusamy et al. Safety

And Efficacy of

Pharmacological Approaches

Available for Multisystem

Inflammatory Syndrome in

Children (Mis-C): A Systematic

Review. Inplasy protocol

202220052. doi:

10.37766/inplasy2022.2.0052

Received: 14 February 2022

Published: 14 February 2022

Corresponding author:

Muhammad Hibatullah Romli

mhibatullah@upm.edu.my

Author Affiliation:

Universiti Putra Malaysia.

Support: Data analysis.

Review Stage at time of this

submission: Data analysis.

Conflicts of interest:

None declared.

\section{Safety And Efficacy of Pharmacological Approaches Available for Multisystem Inflammatory Syndrome in Children (Mis-C): A Systematic Review}

Velusamy, Y; Romli, MH; Yubbu, P; Aissvarya, S; Thilakavathy, K4.

Review question / Objective: What are the evidence on safety and efficacy of pharmacological treatments used in MIS-C management?

Condition being studied: Multisystem Inflammatory Syndrome in Children (MIS-C) - In early 2020, rare cases associated with SARS-CoV-2 were reported in children known as Multisystem Inflammatory Syndrome in Children (MIS-C) by the Centers for Disease Control and Prevention. This condition which is also known as Paediatric Inflammatory Multisystem Syndrome (PIMS) was initially presented as Kawasaki disease as it has similar symptoms. However, MIS-C was then defined as a new disease because of several differences in features. This emergence addresses the need for safe and efficient treatment approaches.

INPLASY registration number: This protocol was registered with the International Platform of Registered Systematic Review and Meta-Analysis Protocols (INPLASY) on 14 February 2022 and was last updated on 14 February 2022 (registration number INPLASY202220052).

\section{INTRODUCTION}

Review question / Objective: What are the evidence on safety and efficacy of pharmacological treatments used in MIS-C management?

Rationale: This systematic review is needed to study and summarize all the articles on the most safe and effective management methods in treating MIS-C. It provides a comprehensive summary and view of all the studies conducted in a standardized manner which can be used by practitioners to understand a wholesome treatment in a short period of time.

Condition being studied: Multisystem Inflammatory Syndrome in Children (MIS-C) - In early 2020, rare cases associated with SARS-CoV-2 were reported in children known as Multisystem Inflammatory 
Syndrome in Children (MIS-C) by the Centers for Disease Control and Prevention. This condition which is also known as Paediatric Inflammatory Multisystem Syndrome (PIMS) was initially presented as Kawasaki disease as it has similar symptoms. However, MIS-C was then defined as a new disease because of several differences in features. This emergence addresses the need for safe and efficient treatment approaches.

\section{METHODS}

Search strategy: Terms: children, Multisystem Inflammatory Syndrome in Children (MIS-C), and COVID-19. Electronic databases: Academic Search Complete, CINAHL Plus with Full Text, Cochrane Clinical Answers, Cochrane Database of Systematic Reviews, MEDLINE, and Scopus.

Participant or population: Children less than nineteen years old.

Intervention: Pharmacological treatment for MIS-C.

Comparator: Multisystem Inflammatory Syndrome in Children (MIS-C) / Paediatric Inflammatory Multisystem Syndrome (PIMS).

Study designs to be included: Observational studies, case series, retrospective studies and cross-sectional studies.

Eligibility criteria: Children (less than 19 years old); Multisystem Inflammatory Syndrome In Children (M)IS-C / Pediatric Inflammatory Multisystem Syndrome (PIMS); treatment for MIS-C.

Information sources: Electronic databases (Academic Search Complete, CINAHL Plus with Full Text, Cochrane Clinical Answers, Cochrane Database of Systematic Reviews, MEDLINE, and Scopus) were used for the searching of articles. PubMed, Science Direct were used as information sources to get all the desired articles. Manual searching was done separately from the references of other systematic review papers related to the disease being studied.

Main outcome(s): Outcomes of the review is the safety and efficacy of the treatment used for the multisystem inflammatory syndrome in children (MIS-C) that is being studied. This systematic review will provide a comprehensive standardized summary of the existing treatments practices for MIS-C that is safe and efficient to utilize with strong quality of evidence available. Since, there is no standardized treatment for MIS$C$ and it depends on the clinicians to give treatment prescriptions based on either other medical institutions or Kawasaki Disease (KD) guidelines, this evaluation of safety and efficacy helps in determining the best treatment available.

Data management: All the data were extracted in a spreadsheet document with several sheets in one excel document. Next, all the data extracted will be converted into a word file to be used as appendix as references.

Quality assessment / Risk of bias analysis: Newcastle-Ottawa Scale (NOS) was used to assess the quality of articles. Grading of Recommendations, Assessment, Development, and Evaluations (GRADE) was used to assess the outcome of al the articles. Both these tools will assess the Risk of Bias (Rob) as well.

Strategy of data synthesis: Narrative and descriptive analysis using theme development.

Subgroup analysis: Comparing between recovery and unsuccessful rate according to medication.

Sensitivity analysis: None.

Language: English.

Country(ies) involved: Malaysia.

Keywords: Multisystem Inflammatory Syndrome in Children (MIS-C); Paediatric Inflammatory Multisystem Syndrome 
(PIMS); pharmacological treatment; systematic review.

Contributions of each author:

Author 1 - Yasothaa Velusamy - Author 1 contributed to acquisition, formal analysis, data curation, interpretation of data, writing original draft, writing review and editing, drafting of the manuscript.

Email: yasothaavelusamy2@gmail.com

Author 2 - Muhammad Hibatullah Romli Author 2 proposed the design and methodology, contributed in using software, validation, andvisualization. The author also check the clarity and validity of data extracted, reviewed and editedthe manuscript.

Email: mhibatullah@upm.edu.my

Author 3 - Putri Yubbu - Author 3 proposed the concept, contributed in technical, or material support. The author also check the clarity and validity of data extracted, reviewed and edited the manuscript.

Email: drputri@upm.edu.my

Author 4 - Shankar Aissvarya - Author 4 contributed in the study selection process (title and abstract screening).

Email: aissvaryshankar@gmail.com

Author 5 - Karuppiah Thilakavathy - Author 5 contributed in giving administrative support, and approved the final manuscript. Theauthor also check the clarity and validity of data extracted, reviewed and edited the manuscript.

Email: thilathy@upm.edu.my 